\title{
Systemic Inflammatory Response Syndrome as a Reason for the Multiple Organ Failure in a Postpartum Pre- eclamptic Patient
}

Igor V Lakhno

\begin{abstract}
Background: The increased level of the placental proinflammatory cytokines, vasoconstrictors and lipid peroxidation products is known to be involved in the pathogenetic scenario of pre-eclampsia. Systemic inflammatory response syndrome captures the severity of pre-eclampsia (PE). The opinion about the possible role of infection in the origin and progression of pre-eclampsia is rather unpopular nowadays.
\end{abstract}

Aim: To elucidate the involvement of the infectious agents in the pathogenesis of multiple organ failure syndrome in preeclampsia.

Case report: An unusual case of the prodromal symptoms of eclampsia and systemic inflammatory response syndrome in the postpartum period associated with group B streptococcus infection was reported. But the resistant to the cephalosporins microbial flora was a reason for the progression severity of preeclampsia and puerperal sepsis. Since uterus was the source of sepsis the hysterectomy procedure has given a possibility to avoid general peritonitis and septic shock.

Conclusion: The infectious inflammation could be involved in the pathogenesis of $\mathrm{PE}$ and contribute to the progressive severity of the disease.

Keywords: Hypertension, Infectious diseases, Intensive care Medicine.

How to cite this article: Lakhno IV. Systemic Inflammatory Response Syndrome as a Reason for the Multiple Organ Failure in a Postpartum Pre-eclamptic Patient. J South Asian Feder Obst Gynae 2018;10(3):215-217.

Source of support: Nil

Conflict of interest: None

Date of received: $05 / 22 / 2018$

Date of acceptance: 07/05/2018

Date of publication: December 2018

\section{INTRODUCTION}

Pre-eclampsia (PE) is one of the main reasons for maternal morbidity and mortality. Atherosclerosis, hypertension,

\footnotetext{
${ }^{1}$ Associate Professor

${ }^{1}$ Department of Perinatology, Obstetrics and Gynecology, Kharkiv Medical Academy of Postgraduate Education, Kharkiv, Ukraine

Corresponding Author: Igor V Lakhno, Associate Professor, Department of Perinatology, Obstetrics and Gynecology, Kharkiv Medical Academy of Postgraduate Education, Kharkiv, Ukraine, e-mail: igorlakhno71@gmail.com
}

coronary heart disease and brain stroke are the consequences of PE in the further lifetime. ${ }^{1-3}$ This gestational pathology is associated with arterial hypertension, proteinuria, and end-organ malfunction. The placental ischemic syndrome (PIS) was proved to be the initial stage of the systemic vasculopathy in PE. Increased levels of the placental pro-inflammatory cytokines, vasoconstrictors and lipid peroxidation products are known to be involved in the pathogenetic scenario of PE. An endothelial dysfunction is the central event in the end-organ failure $^{4-6}$ The systemic inflammatory response syndrome (SIRS) reflects the severity of PE but it mostly has no clinical equivalents in mild and moderate pre-eclamptic patients. ${ }^{7}$ The SIRS accompanies severe PE or eclampsia and different types of shock. Thus, non-infectious inflammation is a major component of PE. A possible reason of this inflammatory response in the second half of gestation could be associated with the HLA-incompatibility between the mother and the fetus, the fetal DNA, the products of ischemic necrosis or apoptosis of the syncytiotrophoblast etc..$^{3-5,8}$ The lack of the evidence makes the possible role of infection in the origin and progression of PE rather unpopular. ${ }^{8}$ A higher incidence of PE occurs in pregnant women with urinary tract infection, periodontal disease, bacterial vaginosis and other comorbidities. ${ }^{9-12}$ Any maternal infection was associated with two-fold higher risk of PE in one systematic review. ${ }^{11}$ The relevant issue is whether infectious agents are involved in the pathogenesis of multiple organ failure syndrome in PE.

\section{CASE PRESENTATION}

A pregnant woman aged 30 years was admitted to the division of maternal-fetal medicine at 24 weeks of gestation. She had had irregular antenatal visits, which revealed no abnormality. She had a blood pressure (BP) of $140 / 90$, pulse of 82 per minute and a normal body temperature at the admission office. She had no prodromes suggestive of arterial hypertension. Laboratory findings were unremarkable (hemoglobin, leukocytes, platelet count, serum aspartate aminotransaminase, serum alanine aminotransaminase, serum urea, serum creatinine concentration and coagulation profile were normal) with a trace of proteinuria in urinalysis. A bacterial vaginosis was diagnosed. She had a mild PE, an 
early fetal growth restriction and a post-cesarean uterine scar. No maternal internal diseases were known. The maternal condition was stable but fetal demise occurred. Ceftriaxone injections were started, as is our standard practice for intrauterine death. Labor was induced by an intracervical insertion of laminaria. A dead female fetus of $510 \mathrm{~g}$ was born. A curettage was performed because of the uterine scar and the partly retained placenta. The patient additionally received $2 \mathrm{~g}$ of ceftriaxone and 500 mg of metronidazole intravenously. The BP was 130/95, urinary protein in a single portion- $0.015 \mathrm{~g} / \mathrm{L}$, hematological indices were normal. The postpartum woman complained of headaches, visual disturbances and mild muscles spasms within 8 hours. BP consequently jumped to $210 / 130$, proteinuria to $6.5 \mathrm{~g} / \mathrm{L}$. These data and the absence of any internal disease in the history supported severe PE. She did not have any generalized seizures. The patient received $4 \mathrm{~g}$ of $\mathrm{MgSO} 4$ within 20 minutes intravenously as a loading dose and then an infusion of $\mathrm{MgSO} 4$ was maintained at a rate of $1 \mathrm{~g}$ / hour for 24 hours. An infusion of $60 \mathrm{mg}$ of hydralazine was also done at a rate $2-10 \mathrm{mg} /$ hour depending on the $\mathrm{BP}$ and pulse rate. BP fell to 150/90. The data of the bio-impedance cardiography supported the hypokinetic type of the central hemodynamics with end-organ hypoperfusion. The patient received antihypertensives, antimicrobial agents, and an infusion therapy. An enlarged hepatic volume was found at the end of the first day of the postpartum period, but the level of serum aspartate aminotransaminase and serum alanine aminotransaminase was not increased considerably and the platelet count was stable. Therefore, HELLP syndrome was excluded. Later on, within 1 day acute renal failure developed (serum creatinine concentration was $2.2 \mathrm{mg} / \mathrm{dL}$ ). A positive test for procalcitonin, an increased level of the C-reactive protein and a leukocytosis were detected at the same time. Therefore, the patient had puerperal sepsis. The uterus was enlarged and painful, the discharge was bloody and purulent. The microbiological investigation of the cervical culture revealed a significant growth of group $B$ streptococcus with the only determined sensitivity to vancomycin. Any agents of sexually transmitted diseases were not detected by polymerase chain reaction. Blood and urine cultures were negative. Rebound tenderness was found in the lower abdomen. Since endometritis and pelvic peritonitis were diagnosed the patient was operated on. A hysterectomy was performed on the 3rd day after delievery. After several days, the condition of the postpartum patient improved. The level of BP was reduced within 10 days. She was back to home in 14 days. A histological investigation supported the purulent endometritis.

\section{DISCUSSION}

Bacterial vaginosis is associated with a higher incidence of the chronic endometritis. ${ }^{9}$ The abnormal uteroplacental hemodynamics caused by group B streptococcusassociated endometritis possibly played a trigger role in the development of PE in this case. Since shallow trophoblastic invasion into the spiral arteries is known as a reason of placental ischemic syndrome, the worsening PE development could be associated with the microbial inhabitants of the genital tract. ${ }^{9,12}$ The problem of resistance to antimicrobial agents contributed to the dramatic outcome. PE typically reduces but not progresses after pregnancy is terminated ${ }^{1-3}$ - our case is unusual in that PE progressed postpartum, possibly related to the inflammatory environment. The decreased intraabdominal pressure after delivery is known to be one of the most significant factors contributing to the reestablishment of hemodynamics. ${ }^{13,14}$ The application of ceftriaxone and metronidazole was ineffective and could not counteract the dissemination of infection after curettage. The clinical manifestation of prodromal events of eclampsia reflected the SIRS. Therefore, the infection likely enhanced the severity of PE and contributed to the development of multiple organ failure syndrome. Since the uterus is the source of sepsis, the hysterectomy enables avoiding a general peritonitis and a septic shock. ${ }^{2,7-13}$ The consequent recovery was rather logic.

\section{CONCLUSION}

The infectious inflammation could be involved in PE pathogenesis and contribute to the progressive severity of the disease even in the puerperium.

\section{DECLARATIONS}

Ethics approval and consent to participate.The study protocol for the reported case was approved by the Bioethics Committee of the Kharkiv Medical Academy of Postgraduate Education (registration number 0105U002865). The patient gave her written consent.

Consent for publication: The patient gave her written consent for publication.

Availability of data and materials: The datasets used during the current study are available from the corresponding author on reasonable request.

Competing interests: The aouthor declares that he has no competing interests.

Authors' contributions: I'm the only author of the paper. 


\section{ACKNOWLEDGMENTS}

Author would like to express his greatest appreciation to Doctor-in-Chief SM Korovay, Prof OV Grishchenko, Prof VG Karpenko, Dr OL Chernyak, Dr IG Polegaeva and Dr VI Iyevleva.

\section{REFERENCES}

1. Hladunewich M, Karumanchi SA, Lafayette R. Pathophysiology of the Clinical Manifestations of Preeclampsia. Clin J Am Soc Nephrol 2007 May;2:543-549.

2. Rosser ML, Katz NT. Preeclampsia: an obstetrician's perspective. Adv Chronic Kidney Dis 2013 May;20(3):287-296

3. UzanJ, Carbonnel M, Piconne O, Asmar R, Ayoubi JM. Preeclampsia: pathophysiology, diagnosis, and management. Vasc Health Risk Manag 2011 Jul;7:467-474.

4. Friedman AM, Cleary KL. Prediction and prevention of ischemic placental disease. Semin Perinatol 2014 Apr; 38(3): 177-182.

5. Elliot M. G. Oxidative stress and the evolutionary origins of preeclampsia. J Reprod Immunol 2016 Apr;114:75-80.

6. Wang PH, Yang MJ, Chen CY, Chao HT. Endothelial cell dysfunction and preeclampsia.J Chin Med Assoc. 2015 Jun;78(6):321-322.
7. Daneva AM, Hadži-Lega M, Stefanovic M. Correlation of the system of cytokines in moderate and severe preeclampsia. Clin Exp Obstet Gynecol 2016 March;43(2):220-224.

8. Ann-Charlotte I. Inflammatory mechanisms in preeclampsia. Pregnancy Hypertens. 2013 Apr;3(2):58.

9. Lopez-Jaramillo P, Herrera JA, Arenas-Mantilla M, Jáuregui IE, Mendoza M A. Subclinical infection as a cause of inflammation in preeclampsia. Am. J. Ther. 2008 Jul-Aug;15(4):373376.

10. Conde-Aqudelo A, Villar J, Lindheimer M. Maternal infection and risk of preeclampsia: systematic review and metaanalysis. Am J Obstet Gynecol. 2008 Jan; 198(1): 7-22.

11. Rustveld LO, Kelsey SF, Sharma R. Association between maternal infections and preeclampsia: a systematic review of epidemiologic studies. Matern Child Health J. 2008 Mar; 12(2):223-242.

12. Herrera J. A., Chaudhuri G., Lopez-Jaramillo P. Is infection a major risk factor for preeclampsia? Med. Hypotheses. 2001 Sep;57(3):393-397.

13. Sugerman H. J. Hypothesis: preeclampsia is a venous disease secondary to an increased intra-abdominal pressure. Med Hypotheses. 2011 Nov;77(5):841-849.

14. Maeda K. Preeclampsia is caused by continuous sympathetic center excitation due to an enlarged pregnant uterus. J Perinat Med. 2014 Mar;42(2):233-237. 